\title{
Study of the relationship of the changes of cervical MRI ,Tran cranial duplex and Brain stem auditory evoked potential in patients with isolated vertigo N.A.Metwally ${ }^{1}$, K.O.Mohammed ${ }^{2}$, M.M.ELmoursy ${ }^{3}$, A.N.Abdelal ${ }^{1}$ and A.F.Elhadad ${ }^{1}$ \\ ${ }^{1}$ Neurology, Dept., Faculty of Medicine, Al-Azhar Univ., Assuit, Egypt \\ ${ }^{2}$ Neurology, Dept., Faculty of Medicine, Assuit Univ., Assuit, Egypt \\ ${ }^{3}$ Audio vestibular unit, E.N.T , Dept., Faculty of Medicine, Al-Azhar Univ., Assuit, Egypt \\ E-mail:dr. AbdelrahmanNoureldeen@yahoo.com
}

\begin{abstract}
Background: Vertigo is an illusory sense of motion in the absence of real movement, either in the self or the environment. Vertigo is isolated and has no positive indications of dysfunction of the central neurological system. Study aims at studying MRI, TCD and BAEP relationships and alterations in individuals with isolated vertigo. Methods: Patient control research involves 50 patients with isolated vertigo, 50 matched age and gender health controls, from Al-Azhar University Hospitals, Assuit, Egypt, neurology and audio vestibular hospitals, from May 2019 through to June 2021. Results: patient start age with vertigo (40-60), disease duration (2-3) years, 70\% with peripheral vertigo, $30 \%$ with central vertigo, $86 \%$ with abnormal and $70 \%$ with abnormal BAEP and $74 \%$ with vertigo with an abnormal TCD. Relationship and variations in cervical MRI, TCD and BAEP in 50 patients with "isolated" vertigo and 50 healthy controls respectively were examined. Results: A statistically significant differences between the two groups were found for total TCD, BAEP abnormalities and no significant differences between the two groups in cervical MRI abnormalities. The findings revealed strong associations between TCD and cervical MRI, TCD and BAEP in the vertigo group. And TCD PI analysis and certain BAEP items revealed positive linear relationships. In the control group, there were no statistical differences or relationships. Conclusions: TCD is a "isolated" vertigo screening sensitive technique. A combined cervical MRI, TCD and BAEP test regimen is suited to evaluate the "isolated" vertigo.
\end{abstract}

Keywords: TCCD, BAEPS, PI, MFV, MRI.

\section{Introduction}

Vertigo is not a single illness but a symptom of a broad variety of illnesses of different aetiology, which may originate in the inner ear, brain and brain stem or can be of internal, vestibular, or psychosomatic origin [1].

Isolated vertigo seems more complicated if both neurological testing and MRI are negative. [2]

Kerber et al (2006)[3] found that individuals with an isolated vertigo symptom had a modest stroke prevalence $(0.7 \%)$, while patients with mixed vertigo symptoms and unbalance have a significant incidence of stroke (3.2 percent ).

Nearly 50 percent of dizzy individuals have vertigo $[4,5]$.

The vertigo of central neurological vascular origin should be distinguished from benign peripheral diseases at an early stage after the development of symptoms [6].

Dizzy individuals are frequently wrongly diagnosed with VBI if they fall within a category of stroke-prone age, thus errors in diagnosis and inadequate treatment of stroke are ordered[7].

Vertebrobasilar arteries supply the key components of the vestibular system with arterial blood flow (inner ear, VIII nerve, and vestibular nuclei and their connections with folliculonodular lobes of the cerebellum). It is thus frequent that decreased blood flow may lead to vertigo in the vertebrobasilar system Hypo-perfusion or transitory ischemia may accompany brief symptoms but more severe symptoms may be caused to post- fossalcirculation thrombotic vascular accidents [8].
Cervical vertigo may or may be one of the major causes of vertigo [9].

The Audio Brain Stamm Reaction (ABR) or Audio Evoked Potentials (BAEPs) is a straightforward, objective and non-invasive approach utilised in the assessment of the brainstem's cochlear nervous and auditory pathways [11].

In addition, ABR may help to locate lesion locations and differentiate between vertigo related to vestibular dysfunction owing to VBI and vertigo. VBI presence affects the integrity of the cochlear nerve vestibular and brain stem that may be identified with the use of ABR [11].

Transcranial Doppler (TCD) is a cheap, painless and non-invasiv technique for measuring the rate and direction of blood flow from the vertebral, basilar, extra- and intracranial sections of the artery $[12,13$, and 14].

The use of (TCD) and (TCCD) in vertigo patients has many advantages: they may be used repeatedly in the nurse of the patient and also cheaply. It may assist differentiate an ischemic stroke from a hemorrhagic stroke by an active stroke with an expert physician [15].

Olszewski et al (2006) [16] suggest TCD for cervical vertigo diagnosis. In a TCD examination with a 15 percent reduction in vertebral artery flux, the presence of a positive neck rotation test indicates the cervical vertigo.

\section{Patients and methods.}

This study was a case-control study. Included 50 patients with isolated symptom of vertigo recruited 
from 312 patients with vertigo, 50 healthy individuals of matched age and sex from Neurology and audio Vestibular unit ENT departments.

The study was approved by the ethics committee of research involving human subjects of faculty of medicine Al- Azhar University, Assuit branch. Informed consent was obtained from each individual before being enrolled in the study.

\subsection{All patients were subjected to}

1. Full medical, audio vestibular, neurological history and examination.

2. Blood tests: Including (CBC, ESR, CRP, Fasting blood sugar, 2hour post prandial blood sugar, Liver function tests (AST, ALT), Kidney function tests (Serum creatinine and urea), Thyroid function tests(Free T3, Free T4 and TSH).

3. Cervical MRI: was acquired on a $1.5 \mathrm{~T} \mathrm{MR}$ Scanner (Philips, Netherland), Conventional turbo spin echo T1-and T2-weighted sagittal and axial images were obtained.

4. Transcranial Duplex: The apparatus used in this study is Philips ClearVue 650 Ultrasound System with L 12-3 MHz linear transducer probe.

\subsection{Statistical analysis}

The collected data was revised, coded, tabulated and introduced to a PC using Statistical package for Social Science (IBM Corp. Released 2017. IBM SPSS Statistics for Windows, Version 25.0. Armonk, NY: IBM Corp.). Data were presented and suitable analysis was done according to the type of data obtained for each parameter. Descriptive statistics: Mean Standard deviation ( \pm SD) for numerical data. Frequency and percentage of nonnumerical data. Shapiro test was done to test the normality of data distribution. Analytical statistics: Student $\mathrm{T}$ Test was used to assess the statistical significance of the difference between two study group means.

\section{Results}

The present study included 50 patients with isolated vertigo and 50 healthy control subjects of matched age and sex.

In vertigo group, cervical MRI results showed normality in 16 cases, cervical curvature abnormality in 5 cases, intervertebral space narrowing in 4 cases, cervical disk bulging in 20 cases, and cervical disk herniation in 5 cases. In control group, cervical MRI results showed normality in 20 cases, cervical curvature abnormality in 30 cases. There was no significant difference of cervical MRI between two groups and the results showed significant differences of TCCD and BAEP between two groups. Table (1)

There is significant differences of Mean flow velocity (MFV) findings of Right vertebral V2 segment, BA, Left V2 a between patients and control group.

There is significant differences of Pulsatility index (PI) findings of basilar between patients and control groups, with no significant differences of PI of right and left V2 Table (2).

Table (1) the overall abnormalities of three examinations of the studied patients and controls groups.

\begin{tabular}{lccccc}
\hline & Cases & $\mathbf{N}=\mathbf{5 0}$ & Controls & $\mathbf{N = 5 0}$ & P-Value \\
\hline Cervical MRI & $\mathbf{N}$ & $\mathbf{( \% )}$ & $\mathbf{N}$ & $\mathbf{( \% )}$ & \\
Normal & 16 & 32.0 & 20 & 40.0 & 0.53 NS \\
Abnormal & 34 & 68.0 & 30 & 60.0 & \\
TCCD & $\mathbf{N}$ & $(\boldsymbol{\%})$ & $\mathbf{N}$ & $(\boldsymbol{\%})$ & \\
Normal & 13 & 26.0 & 40 & 80.0 & $<0.05 \mathrm{~S}$ \\
Abnormal & 37 & 74.0 & 10 & 20.0 & \\
BAEP & $\mathrm{N}$ & $(\%)$ & $\mathrm{N}$ & $(\%)$ & \\
Normal & 15 & 30.0 & 48 & 96.0 & $<0.05 \mathrm{~S}$ \\
Abnormal & 35 & 70.0 & 2 & 4.0 & \\
\hline
\end{tabular}

Table (2) Mean flow velocity (MFV) and Pulsatility index (PI) findings of vertebral and basilar arteries between the studied patients and controls groups.

\begin{tabular}{lccccc}
\hline & Cases & $\mathbf{N = 5 0}$ & Controls & $\mathbf{N = 5 0}$ & P-Value \\
\hline $\begin{array}{l}\text { Parameters } \\
\text { Right }\end{array}$ & Mean & $\mathbf{\pm S D}$ & Mean & $\mathbf{\pm S D}$ & \\
RV2 MFV & 32.22 & 7.19 & 24.73 & 7.16 & $<0.05 \mathrm{~S}$ \\
RV2 PI & 1.05 & 0.2 & 1.12 & 0.24 & $>0.05 \mathrm{NS}$ \\
BA PI & 1.06 & 0.25 & 0.92 & 0.16 & $<0.05 \mathrm{~S}$ \\
Left & & & & & \\
LV2 MFV & 17.11 & 5.83 & 27.35 & 9.4 & $<0.05 \mathrm{~S}$ \\
LV2 PI & 2 & 0.62 & 1.1 & 0.22 & $>0.05 \mathrm{NS}$ \\
LV2 MFV & 17.11 & 5.83 & 27.35 & 9.4 & $<0.05 \mathrm{~S}$ \\
LV4 PI & 1.13 & 0.29 & 0.9 & 0.24 & $<0.05 \mathrm{~S}$ \\
\hline
\end{tabular}


ABR study revealed that 35 patients $(70 \%)$ have abnormalities in one or more parameters of ABR .There were statistically significant differences of I, III, V latency on the left side, and also I, III latency and III-V IPL on the right side between two groups. Table (3)
There were statistically significant differences between BA PI and III-V IPL, I-V IPL on the left side with no statistically significant differences between BA PI and BAEP on the right side. Table (5)

Table (3) BAEP latency and IPL between vertigo and control groups.

\begin{tabular}{|c|c|c|c|c|c|}
\hline & $\begin{array}{r}\text { Cases } \\
\text { Mean } \\
\text { Right BAEP }\end{array}$ & $\begin{aligned} & \mathrm{N}=50 \\
& \pm \mathrm{SD}\end{aligned}$ & $\begin{array}{r}\text { Controls } \\
\text { Mean }\end{array}$ & $\begin{aligned} & \mathbf{N}=50 \\
\pm \mathrm{SD} & \end{aligned}$ & P-Value \\
\hline I & 2.24 & 0.48 & 1.35 & 0.19 & $<0.05 \mathrm{~S}$ \\
\hline III & 4.34 & 0.53 & 3.26 & 0.47 & $<0.05 \mathrm{~S}$ \\
\hline V & 5.44 & 0.56 & 5.35 & 0.36 & $>0.05 \mathrm{NS}$ \\
\hline I-III & 2.016 & 0.4 & 2.09 & 0.23 & $>0.05 \mathrm{NS}$ \\
\hline III-V & 1.72 & 0.44 & 1.67 & 0.14 & $<0.05 \mathrm{~S}$ \\
\hline I-V & 3.99 & 0.45 & 3.9 & 0.29 & $>0.05 \mathrm{NS}$ \\
\hline \multicolumn{6}{|c|}{ Left BAEP } \\
\hline I & 2.25 & 0.48 & 1.39 & 0.22 & $<0.05 \mathrm{~S}$ \\
\hline III & 4.36 & 0.51 & 3.25 & 0.54 & $<0.05 \mathrm{~S}$ \\
\hline $\mathbf{V}$ & 6.39 & 0.53 & 5.38 & 0.42 & $<0.05 \mathrm{~S}$ \\
\hline I-III & 2.18 & 0.39 & 2.11 & 0.24 & $>0.05 \mathrm{NS}$ \\
\hline III-V & 1.69 & 0.44 & 1.67 & 0.15 & $>0.05 \mathrm{NS}$ \\
\hline I-V & 4.1 & 0.45 & 3.87 & 0.28 & $>0.05 \mathrm{NS}$ \\
\hline
\end{tabular}

Table (4) Performance of TCD and BAEP for diagnosis of vertigo using ROC Curve.

\begin{tabular}{llllll}
\hline Markers & Cutoff & AUC & $\mathbf{( 9 5 \%}$ CI) & Sensitivity (\%) & Specificity (\%) \\
\hline BAEP & 3.01 & 0.74 & $(0.64-0.82)$ & $74 \%$ & $92 \%$ \\
TCD & 1.25 & 0.58 & $(0.48 * 0.68)$ & $90 \%$ & $82 \%$ \\
\hline
\end{tabular}

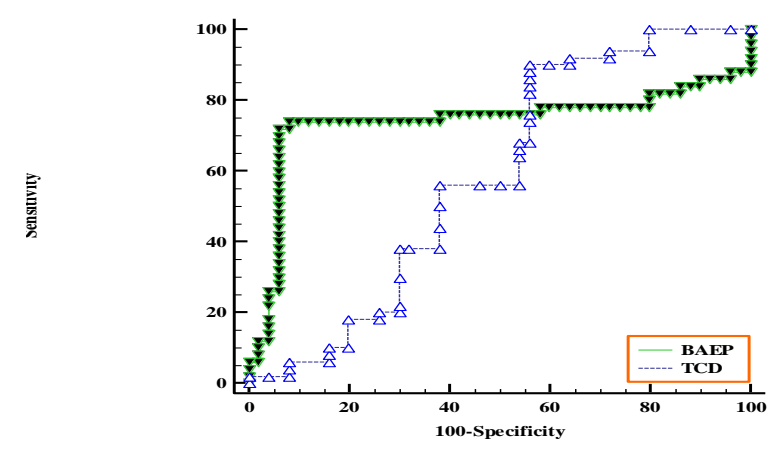

Fig. (1) ROC Curve of TCD and BAEP for diagnosis of vertigo

Table (5) Correlation between Basilar pulsatility index (PI) and BAEP parameters between the studied cases.

\begin{tabular}{llcc}
\hline & RT BAEP & r- Value & P-Value \\
\hline BA PI & I & 0.011 & $>0.05 \mathrm{NS}$ \\
& III & -0.031 & $>0.05 \mathrm{NS}$ \\
& V & 0.025 & $>0.05 \mathrm{NS}$ \\
& I-III & -0.009 & $>0.05 \mathrm{NS}$ \\
& III-V & -0.011 & $>0.05 \mathrm{NS}$ \\
& I-V & -0.006 & $>0.05 \mathrm{NS}$ \\
\hline BA PI & LT BAEP & r- Value & P-Value \\
& I & 0.011 & $>0.05 \mathrm{NS}$ \\
& III & -0.015 & $>0.05 \mathrm{NS}$ \\
& I-III & 0.023 & $>0.05 \mathrm{NS}$ \\
& III-V & -0.013 & $>0.05 \mathrm{NS}$ \\
& I-V & -0.32 & $<\mathbf{0 . 0 5} \mathbf{S}$ \\
\end{tabular}




\section{Discussion}

Isolated vertigo seems to be more Complex, if neurological tests are negative for both neurological and head RMR [2]. Certain vertigo may occur in brain stem or cerebellum, which has a significant effect on the patient [2].

This research includes 50 solitary vertigo patients, 30 men and 20 females $(53,06 \pm 8)$ years of age. At the same time, there was a recruitment of 50 healthy con trolles matched in sex and age, including 30 males and 20 females, with a mean age $(53.3 \pm 8.01)$ years.

In our research, there is no statistically significant difference between vertigo and control group of cervical MRI abnormalities. It may be ascribed to the significant instances of middle age. The findings are consistent with $\mathrm{Ji}$ and Zhang (2014)[17] which showed that the cervical anomalies of the patients and control groups were not statistically significant.

In our findings the major BAEP anomalies revealed that the delay of I, III and V on the left was statistically significant.

and I, III latency and III-V Interpeak latency between patient and control group on the right-hand side. These findings are consistent with $\mathrm{Ji}$ and Zhang, (2014)[17] who have found statistically significant difference between the two categories I, III, V on the left and I, III latency and III-V on the right.

The results of the study indicated that the statistically significant difference between the patient and the control group was between the median flow velocity (MFV) of the right and left vertebrals (Proximal-Extra cranial (V1); also there were significant differences between the right and left vertebral arteries (PI) findings of pulsatility index (PI) and the control group with. There are also substantial variations between the Pulsatility Index (PI) of the results of Rt and Lt vertebral (V1) between patients and control groups under this agreement with Ji and Zhang, (2014) [17], who found statistical significant differences in (MFV).

A statistically significant difference between patients and the control group was found in Mean Flow Velocity (MFV) of the right and left vertebral (Distal-intracranial (V4). These findings are consistent with Rageh et al. (2010)[18], which showed statistically significant variations in the MFVs of the right and left vertebral arteries between two groups.

The pulsation index (PI) data from Right and Left Vertebral (Distal-intracranial (V4)) between patients and the control group were statistically significant. and These findings accord with Rageh et al., (2010)[18] who found that the right- and leftvertebral artery PI group had statistically significant differences.

The findings revealed that the mean flow-speed (MFV) and the pulsatility index (PI) of basilar arteries were statistically significantly different among patients and the control group. This was agreed with $\mathrm{Ji}$ and Zhang,[17] and Rageh et al.,[2010][18], which reports that the difference between basillary artery parameters in patients and the control group is statistically significant.

In the vertigo group statistically relevant correlation exists between the general abnormalities TCD and cervical MRI $(\mathrm{X} 2=4.77, \mathrm{P}<0.05)$ and TCD and $\mathrm{BAEP}(\mathrm{X} 2=40.77, \mathrm{P}<0.05)$, but there has been no significant correlation between cervical MRI and $\operatorname{ABR}(\mathrm{X} 2=3.43, \mathrm{P}>0.05)$, therefore, cervical MRI and BAEP abnormen in the presence of TCD abnormalities can be predicted.

This result suggests the superiority of the TCD in patients with vertigo over cervical MRI and ABR. This was in agreement with Ji and Zhang (2014)[17], who reported that the correlation was statistically significant not only between TCD and cervical MRI $(\mathrm{X} 2=15.90, \mathrm{p}<0.01)$ but also between TCD and BAEP $(\mathrm{X} 2=17.31,<0.01)$, but also between cervical MRI and BAEP $(\mathrm{X} 2=3.01, \mathrm{p}>0.05)$ and, also, Mohammed et al. (2012)[19] and we could draw the conclusions from this report. The results are not statistically significant.

Our findings also revealed substantial quality correlations between LV1 PI and I, III latency, III-V, I-V IPL on the left and V latency, III-V IPL on the right hand side, apart from the quantitative connection between TCCCD and BAEP. And also there is a correlation between RV1 PI and I, III latency, III -V, I-V IPL to the left, and III-V IPL to the right, and also, to the left, BA PI and III-V, I-V IPL to the left, and also positive linear linkages exist between LV4 PI and V latency, III-V IPL to the left, and I latency to the right. And there was also a correlation between the right latency of RV4 PI and III, V with no links on the left, which confirms that the TCCD and BAEP parameters can reflect brain stem blood supply and nerve function respectively and therefore can distinguish vertigo of vertebrobasilar origin from the periphery. It showed if a patient with isolated vertigo has an abnormal TCD result, we should consider the abnormal BAEP outcomes, and vice versa if a patient with isolated vertigo has an abnormal BAEP result, consider the abnormal TCD results and our current VBI sensitivity outcomes of 90 percent and 82 percent support the clinical value of using TCD.

TCCD and BAEP should thus be investigated in vertigo patients, and TCCD should be the initial evaluation when evaluating vertebrobasilar vertigo. In contrast the combination of cervical MRI, TCD and BAEP has superiorities to exclude vertebrobasilar insufficiency of its negative correlations, with the agreement of Searls et al.,[2012].[2] They have reported that TCD is superior in early detection of isolated vertigo, small vascular focal, neurological injury or other conditions He considered that TCD MFV of vertebral artery declines 15 per cent for positive neck rotation for cervical vertigo diagnosis, and also for Syme $\mathrm{P}$ diagnosis, (2004)[21] reported that TCD is more sensitive (96 per cent) for the early detection of acute occlusions of the small vessel, especially when conducted with transcranial, color-coded duplication. The surveillance of TCD basilar arteries by patients 
with acute post-circulation ischemia was conducted by imaging[2013] and Hwang etc., (2012)[23], and the link was discovered to be between microembolic signals and intracranial vertebrobasillary stenosis It indicated that the post-circulation micro-embolics during the TCD predicted stroke incidence in this region if there was no intervention. TCD should thus be utilised for primary prevention of post-circulation stroke following a vertigo episode.

The total abnormality in vertigo group was $70 \%$ in our research, while only 4 percent in the control group and the lack of head MRI may suggest microvascular ischemia within the imaging threshold compared to Cai et al., (2012)[24] and Grad et al., (1989)(25) in those patients with abnormal BAEP.

5.Conclusion.

TCCD is an essential technique of diagnosing vertigo, a sensitive approach for vertigo screening, and superior to early identification of isolated vertigo, tiny vascular focal cell, neurological injury or other non-structural head damage problems.

The TCCD and BAEP characteristics may represent the status of the brainstem's blood supply and nerve function and can differentiate vertebrobasilar vertigo from peripheral aetiology. It showed that if a patient with isolated vertigo has an abnormal TCD, an abnormal BAEP result should be taken into account and vice versa.

In the case of TCD abnormalities, cervical MRI and BAEP abnormalities may be anticipated.

This result shows that the TCD is superior to cervical MRI and ABR in vertigo patients.

\section{References}

[1] Kadanka, Zdenek, Jura, Rene, \& Bednarik, Josef. Vertigo in Patients with Degenerative Cervical Myelopathy. Journal of Clinical Medicine.vol.10(11),pp.2400-2496,2021.

[2] Searls, D.Eric, Pazdera, Ladislav, Korbel, Evzen, Vysata, Oldrich, \& Caplan, R.Louis Symptoms and signs of posterior circulation ischemia in the new England medical center posterior circulation registry. Archives of neurology.vol.69(3),pp.346-351,2012.

[3] Kerber, A.Kevin, Brown, L.Devin, Lisabeth, D.Lynda, Smith, A.Melinda, \& Morgenstern, B. Lewis Stroke among patients with dizziness, vertigo, and imbalance in the emergency department: a population-based study. Stroke.vol.37(10),pp.2484-2487,2006.

[4] Yacovino, A.Dario, \& Hain, C.Timothy Clinical characteristics of cervicogenicrelated dizziness and vertigo. Paper presented at the Seminars in neurology.vol.15,pp.3000-3200,2013.

[5] Tarnutzer, A.Alexander, Berkowitz, L.Aaron, Robinson, A.Karen, Hsieh, YuHsiang, \& Newman-Toker, David E. Does my dizzy patient have a stroke? A systematic review of bedside diagnosis in acute vestibular syndrome. CmAJ.vol.183,pp. 571-592,2011.
[6] K-D Choi, , H.Lee, , \& J-S.Kim.Ischemic syndromes causing dizziness and vertigo. Handbook of clinical neurology.vol.137,pp.317-340,2016.

[7] Caplan, LR. Transient vertigo, drop spells ، and cerebrovascular disease. Vestibular Disorders. Year Book Medical Publishers.vol17,pp.254-274,1988.

[8] Shepard, T.Neil, \& Telian, A.Steven Practical management of the balance disorder patient: Singular.vol.17,pp800$890,1996$.

[9] Cherchi, Marcello. Epidemiology of dizziness. Journal of Biological Physics and Chemistry, .vol.13,pp.18-29,2013.

[10] OA.Somefun, OS.Giwa, BA.Bamgboye, I.Okeke-Igbokwe, Irene, \& Azeez, AA.Abdul. Vestibular disorders among adults in a tertiary hospital in Lagos, Nigeria. European archives of oto-rhinolaryngology.vol.267(10),pp.1515$1521,2010$.

[11]Rosa, Luana Araujo Cruz, Suzuki, Marcia Rumi, Angrisani, Rosanna Giaffredo, \& Azevedo, Marisa Frassom. Auditory Brainstem Response: reference-values for age. Paper presented at the CoDAS.vol14,pp230-250, 2014

[12] Rincon, Fred, Sacco, L.Ralph, Kranwinkel, Xu.Grace, , Qiu, Paik, C.Myungee, BodenAlbala, Bernadette, \& Elkind, SV.Mitchell Incidence and risk factors of intracranial atherosclerotic stroke: the Northern Manhattan Stroke Study. Cerebrovascular diseases.vol.28(1),pp.65-71,2009.

[13] Aaslid, Rune, Markwalder, Thomas-Marc, \& Nornes, Helge.Noninvasive transcranial Doppler ultrasound recording of flow velocity in basal cerebral arteries. Journal of neurosurgery.vol. 57(6),pp.769-774,1982.

[14] Malferrari, Giovanni, Pulito, Giuseppe, Pizzini, Attilia Maria, Carraro, Nicola, Meneghetti, Giorgio, Sanzaro, Enzo,Monaco, DanielaMicroV technology to improve transcranial color coded Doppler examinations. Journal of Neuroimaging.vol.28(4),pp.350-358,2018.

[15] Siniscalchi, Antonio, \& Malferrari, GiovanniThe roleof transcranial Doppler ultrasonography in differential diagnosis of vertigo in the Emergency Department. Emergency Care Journal.vol. 15(3),pp.5660,2019.

[16] Olszewski, urek, Majak, Joanna, Pietkiewicz, Piotr, Luszcz, Cezary, \&Repetowski, Marcin. The association between positional vertebral and basilar artery flowlesion and prevalence of vertigo in patients with cervical spondylosis. Otolaryngology-Head and Neck Surgery.vol. 134(4),pp.680-684,2006.

[17]Ji.Wenzhen, \& Zhang, XueqingRelationship of the changes of cervicalMRI, TCD and 
BAEP in patients with "isolated" vertigo. International journal of clinical and experimental pathology.vol.7(8),pp.5171,2014 .

[18]Rageh, A.Tarek, Mohamed, S.Enas, \& Elattar, M.Amal The role of brainstem auditory evoked potentials, electronystagmography, and transcranial Doppler in evaluation of patients with vertigo. Egypt J Neurol Psychiat Neurosurg.vol.47(3),pp.511-518,2010.

[19] Mohamed, S.Enass, Kaf, A.Wafaa, Rageh, A.Tarek, Kamel, F.Nageh, \& Elattar, M.Amal Evaluation of patients with vertigo of vertebrobasilar insufficiency origin using auditory brainstem response, electronystagmography, and transcranial Doppler. International journal of audiology.vol.51(5),pp.379-388,2012.

[20] Haupt ‘F.Walter, Pawlik, Gunter, \& Thiel, Alexander. Initial and serial evoked potentials in cerebrovascular critical care patients.Journal of clinical neurophysiology.vol.23(5),pp.389-394,2006.

[21] Syme, Paul. Detection of small vessel knock using transcranial doppler ultrasonographyimplications for the ischaemic penumbra and the treatment of small vessel occlusive stroke. Adv Clin Neurosci Rehabilitation.vol.4,pp.28-31,2004.

[22] Stayman, Aaron, Nogueira, G.Raul, \& Gupta, RishiDiagnosis and management of vertebrobasilar insufficiency. Current treatment optionsin cardiovascular medicine.vol.15(2),pp.240-251,2013.

[23] Hwang, Jaechun, Kim, Suk Jae, Hong, Ji.Man, Bang, Oh.Young, Chung 'ChinSang, Lee, Ho .Kwang, \& Kim, GyeongMoon.Microembolic signals in acute posterior circulation cerebral ischemia: sources and consequences. Stroke.vol.43(3),pp.747-752,2012.

[24] Cai, Zeng-Lin, Zhang, Ni.Zheng-Chun, JianQiang ‘Xue, Xu.Shou-Ru, Li-Zhen, \& Wu, Fang-PingThe changes of brainstem auditory evoked potentials (BAEP) after vertebrobasilar artery ischemia in rabbits. Neurological Sciences.vol.33(5),pp.1155$1160,2012$.

[25] Grad, Anton, \& Baloh, W.Robert Vertigo of vascular origin: clinical and electronystagmographic features in 84 cases. Archives of neurology.vol.46(3),pp.28128,1989 . 